\title{
Dans les paysages de Motten Morvan : une expérience d'art écologique
}

\author{
In the landscapes of Motten Morvan: an experience of ecological art
}

\author{
Anaïs Belchun ${ }^{1}$ \\ ${ }^{1}$ LARA-SEPPIA, Université Toulouse Jean-Jaurès.
}

\begin{abstract}
RÉSUMÉ. Cet article présente un travail de création-recherche en art, croisant des réflexions sur le paysage et l'écologie. Comment une pensée de l'écologie peut-elle guider des pratiques artistiques et paysagères ? Quel rôle peuvent jouer la recherche universitaire et création artistique dans le partage et le déploiement d'une vision du monde écologique ? Ces interrogations ont été explorées à travers un projet fondé sur une démarche de création-recherche et une démarche de création inductive. Je présente ici les particularités méthodologiques et épistémologiques de ce processus de recherche, à travers des expérimentations concrètes. Je présenterai ensuite les expériences artistiques et paysagères qui ont été mises en œuvre sur le site de Motten Morvan. II s'agit d'abord de créations artistique in situ, sous la forme d'un sentier paysager et la conception d'installations ; ensuite d'actions pédagogiques, à travers des ateliers d'écoformation par les arts du paysage. Cette expérience d'art écologique propose ainsi une piste de réflexion pour comprendre comment l'étude et la pratique des arts du paysage peuvent nous aider à mieux habiter la Terre.

ABSTRACT. This article presents a creation-research work in arts, meeting thoughts about landscape and ecology. How can an ecological thinking guide artistic and landscape practices? What role can academic research and artistic creation play in sharing and deploying an ecological world view? These questions were explored through a project based on a creation-research approach and an inductive approach. Here I present the methodological and epistemological particularities of this research process, through concrete experiments. I will then present the artistic and landscape experiments that have been carried out on the site of Motten Morvan. These are, first of all, in situ artistic creations, in the form of a landscape trail and art installation's design ; then of educational actions, through workshops of ecoformation by the landscape arts. This ecological art experience offers a way to think about how the study and practice of landscape arts can help us to better inhabit the Earth.

MOTS-CLÉS. Art écologique, création-recherche, écoformation, paysage.

KEYWORDS. Ecological art, research-creation, eco-education, landscape.
\end{abstract}

\section{Introduction}

La question d'une approche écologique et sensible du paysage ${ }^{1}$ est au cour de mon travail de recherche. Je prépare actuellement une thèse en arts plastiques intitulée «Écopoétiques paysagères : une pensée de l'écologie à l'œuvre dans les arts du paysage ». J'étudie donc comment l'approche paysagère peut s'instaurer, au croisement d'une pensée de l'écologie et d'une pratique artistique. La problématique guidant cette recherche est issue de deux questions complémentaires: d'une part, comment une vision du monde écologique peut-elle nourrir des pratiques artistiques et paysagères ? D'autre part, quel rôles peuvent jouer les approches paysagères et artistiques dans le développement et le partage de cette pensée écologique ? Cette deuxième question élargit le champ de la recherche en art, en intégrant un objectif pédagogique d'écoformation.

Je vais présenter ici les modalités concrètes de ces recherches. Dans un premier temps, je présenterai le processus de travail, en expliquant l'approche dite de "création-recherche » que j'ai adoptée, et en détaillant la démarche de création inductive qui en découle. Dans un second temps, je

${ }^{1}$ Cet article présente un travail en cours, présenté en février 2019 lors du séminaire de création-recherche « Approches écologiques et sensibles du paysage : approches artistiques " au sein du LARA-SEPPIA, laboratoire de recherche en arts de l'Université Toulouse - Jean Jaurès. 
vous présenterai les expériences artistiques que j’ai menées depuis 2016 sur le site de Motten Morvan, qui comprennent un aménagement et la conception d'installations in situ, ainsi que l'animation d'ateliers d'écoformation. Pour préciser le sens de ce travail par rapport à mes thématiques de recherche que sont le paysage et l'écologie, il me faut d'abord présenter rapidement quelle sont mes approches de ces deux notions.

Que signifie «penser l'écologie»? Cela commence par la mise en question des discours ordinaires sur l'écologie, selon une approche critique de la «fiction verte ». L'écologie peut être reconsidérée à partir de sa définition scientifique, en tant que «science des relations entre les êtres vivants et leur milieu », et la mise en relation avec la pensée systémique permet de mieux en cerner les subtilités. Une pensée de l'écologie émerge dans les domaines philosophiques et politiques, selon deux courants divergents : l'environnementalisme (lié au développement durable) et l'écologie radicale (incluant l'écologie profonde). Des nombreuses réflexions sur l'écologie se déploient aussi dans le domaine des sciences humaines, parmi lesquelles se dessinent des pistes de convergences. Il en émerge une nouvelle forme de pensée, systémique et transdisciplinaire, dont les problématiques peuvent être résumées en deux questions : «Comment vivre dans la communauté du Vivant ? » et «Comment habiter la Terre ? ». Cela implique de développer une forme de respect et de solidarité entre l'ensemble des êtres vivants (humains, animaux, végétaux...), tout en prenant en compte les dynamiques écosystémiques, de l'échelle locale à l'échelle planétaire. Une nouvelle vision du monde émerge de cette pensée écologique, qui peut être rapprochée de la mésologie d'Augustin Berque ou de la géopoétique de Kenneth White. Elle s'exprime aussi sous des formes plus sensibles, littéraires et esthétiques. J'étudie le concept d'écopoétique, pour désigner cette approche sensible de l'écologie, qui évoque le sens que nous donnons au monde selon les expériences relationnelles que nous vivons.

Que peut alors être une approche écopoétique du paysage ? Et d'abord, qu'est-ce-que le paysage ? Cette notion ne désigne pas la même chose pour un géographe, un historien de l'art, un écologue, etc. Je m'intéresse en fait au paysage pour la complexité et la transdisciplinarité que cette notion suppose, en le redéfinissant comme une expérience relationnelle dotée de sens entre un être humain et un milieu. J'analyse selon une approche critique différents modèles paysagers, qui se rattachent à la fiction verte, ou au contraire se dirigent vers une écopoétique paysagère. J'étudie plus précisément leur expression dans les arts du paysage, ce qui désigne différentes formes artistiques, de l'aménagement de site à la représentation visuelle, en passant par la marche ou le land art.

\section{Processus de création-recherche}

\subsection{Un projet de création-recherche}

Ces recherches théoriques que je développe à travers des études d'écrits et d'œuvres ne constituent pas l'ensemble de mon travail de recherche, qui est abordé selon une démarche spécifique de «création-recherche ». Cette approche, qui s'inscrit dans la ligne identitaire de notre laboratoire de recherche, consiste à associer la création artistique à la recherche universitaire, dans un seul et même processus. La recherche en art ainsi conçue ne se limite donc pas à la production d'un discours scientifique sur l'art, par l'étude d'œuvres réalisées par d'autres artistes; elle y associe la recherche par l'art, en intégrant la conception et la réalisation artistique en tant que processus producteur de savoirs.

Cette démarche de création-recherche peut être abordée de multiples manières. Par exemple, elle peut être le fait d'un-e artiste qui s'interroge sur les modalités et les enjeux de sa création, sur le sens qui en émerge, et décide d'entreprendre des recherches pour approfondir ces réflexions. Auquel cas la relation entre la création et la recherche se présente comme une évidence. Il peut aussi s'agir d'un désir de mettre en relation deux activités initialement séparées, de création artistique et de réflexion 
théorique. C'est un tel processus qui a guidé mes toutes premières recherches en master, sur les relations entre la pratique du graphisme et mes idées sur l'écologie. Ce travail a donné lieu à une production graphique et un mémoire intitulé «praxis du graphisme et écologie, un engagement audelà de la fiction verte ». J'ai ensuite décidé d'approfondir ces recherches, en les ouvrant à d'autres pratiques artistiques, autour de la notion de paysage. Mais pour ce travail de thèse, la situation était différente, puisque j'avais peu d'expériences préalables sur lesquelles me fonder. Il m'a donc fallu adopter une autre approche de la création-recherche, qui ne soit pas construite à partir d'une pratique antérieure, mais en vue d'élaborer à la fois une pratique artistique nouvelle - pour moi - et une pensée théorique originale.

Avec cette approche orientée vers un objectif, on entre dans une démarche de projet. La création artistique est alors conçue comme une expérimentation pratique, qui se fonde sur une recherche théorique et la nourrit également. Cette approche par la démarche de projet et par l'expérience m'amène à une vision pragmatique de la recherche et de la création artistique : pragmatique dans le sens philosophique du terme, d'après les idées de John Dewey, qui mettait l'expérience au centre de ses visions de l'art et de la pédagogie. L'expérience sous toutes ses formes est donc une notion centrale de ma démarche de création-recherche, qu'il s'agisse de l'expérience directe et sensible du monde qui en est le point de départ, de l'expérimentation qui est au cœur du processus de recherche, ou encore de l'expérience que je tire de ces pratiques et qui me permet de développer un positionnement théorique sur des bases solides.

Ce travail d'expérimentation, tout à fait concret, ne pouvait se réaliser qu'à partir d'un terrain tout aussi concret. Issue des sciences sociales, la notion de terrain renvoie à un environnement défini par des critères géographiques, temporels et sociaux, qui permet de confronter des hypothèses théoriques à une situation réelle, et sert de support à une enquête, dont les données seront ensuite interprétées pour l'élaboration théorique. Mais pour une artiste, le terrain est plutôt considéré comme l'environnement de création d'une œuvre. Et, dans un travail sur le paysage et l'écologie, on est ramené à la définition littérale du terrain en tant que «portion de terre ». Il s'agit donc d'un lieu défini à la surface de la terre, marqué par ses caractéristiques géographiques - c'est-à-dire ses singularités physiques, écologiques, historiques, sociales, etc. J'ai trouvé ce terrain dont j'avais besoin en rencontrant une association, qui a mis à ma disposition un site de trois hectares en Bretagne pour développer ce projet, ainsi qu'un réseau de relations locales. Ce n'est que lorsque j'ai trouvé ce terrain que j'ai pu développer vraiment mon travail de recherche.

La démarche de projet qui débute alors est marquée par la complexité. Il s'agissait d'abord de prendre en compte en pratique la complexité induite par mes sujets de recherche: l'approche transdisciplinaire impliquée par les thématiques de l'écologie du paysage d'une part, la complexité de la notion même de paysage d'autre part. Ce qui m'a aussi amené à adopter la pensée complexe comme traduction méthodologique de la pensée systémique associée à l'écologie. De plus, la démarche de création-recherche induit aussi sa propre complexité, inhérente aux aller-retours entre la science et l'art et entre la théorie et la pratique. Il faut ajouter la complexité des diverses formes artistiques que j'ai été amenée à expérimenter, in situ et in visu, avec toute l'indétermination impliquée par l'évolution du terrain dans le temps.

Tout cela m'amène à développer une méthodologie adaptée, que je nomme une approche systémique du projet. Cette approche repose sur l'application des grands principes de la pensée systémique : la prise en compte de la complexité, une attention portée sur les relations (plutôt que sur la distinction entre les éléments), et la prise en compte de leur dimension dynamique, évolutive. Cette attention aux relations s'applique d'abord aux relations entre les différentes composantes internes du projet: il s'agit par exemple d'accorder ensemble les questions budgétaires et d'écoconception, d'associer les moyens techniques et humains, etc. Elle s'applique aussi aux relations environnementales, le projet étant conçu et évoluant selon le contexte social, le réseau relationnel, 
l'environnement naturel, d'autres projets existants, etc. La perception de la dimension dynamique du projet implique de prévoir ou du moins d'intégrer au fur et à mesure les évolutions possibles du projet : par exemple selon la progression théorique, une baisse de budget, de nouveaux partenaires, l'évolution de la végétation sur le terrain (enfrichement ou défrichage, arbres abattus)...

La dernière dimension importante de ma démarche de création-recherche est liée à l'orientation écologique de ce projet paysager, avec l'étude de ce que peut être une démarche d'éco-conception artistique et paysagère. L'éco-conception est une notion issue du domaine industriel, désignant une démarche de conception de produits visant à limiter les impacts environnementaux tout au long de son cycle de vie (de la matière première à la fin de vie, en passant par la fabrication, la logistique, la distribution et l'usage). Elle est aussi appliquée à d'autres domaines, notamment ceux de l'architecture ou de l'aménagement paysager. J'estime que cette notion d'éco-conception peut être appliquée plus largement, à tout type de projet. En effet, une vision du monde écologique implique une praxis écologique, qui se traduit dans une démarche de création particulière. J'utilise ici le terme de praxis comme notion philosophique, pour désigner une pratique qui se déploie en relation avec une pensée théorique et selon une posture politiquement engagée. C'est selon cette optique que j'étudie comment l'éco-conception peut être appliquée dans ce projet, en tant que projet de paysage, et en tant que projet artistique. Différentes dimensions du projet doivent alors être prises en compte, selon des paramètres concrets (environnementaux, matériels, humains...), mais aussi culturels (éthique, sens de la réalisation, message communiqué, écopoétique...). Cette démarche d'écoconception m'amène également à prendre en compte les dimensions expérimentales et pédagogiques de l'oeuvre. Mais avant tout, elle implique d'adopter une démarche de création centrée sur les singularités écologiques du terrain investi pour la réalisation du projet.

\subsection{Une démarche de création inductive}

Les spécificités de mes recherches m’entrainent à accorder une grande importance au terrain, pour plusieurs raisons. Sur le plan théorique, la vision du monde écologique et systémique adoptée, ainsi qu'une conception du paysage comme expérience relationnelle entre l'humain et le monde, supposent une attention aux relations - toujours singulières - qui s'établissent entre un être ou une personne et son milieu de vie. Sur le plan pratique, les démarches de projet paysager et d'écoconception impliquent de considérer le terrain choisi, avec toutes ses particularités, comme point de départ du projet. Tout cela nous amène donc à une démarche de création inductive : c'est le terrain qui impulse la conception du projet artistique, en fonction notamment des particularités du site. Ce terrain est en effet avant tout un lieu, c'est-à-dire une portion d'espace telle qu'elle peut être perçue par un être vivant. Cette attention à la perception sensible du lieu est celle d'une expérience esthétique - ce terme provenant du grec aesthesis qui signifie «qui a la faculté de sentir, sensible, perceptible ». On pourrait donc penser que cette démarche inductive est dirigée vers une esthétique du lieu. Mais mon approche écologique du paysage m'amène à aller au-delà de la notion de lieu, en considérant comment la relation entre un lieu et un être vivant devient pour lui signifiante, et par là constitutive de son monde propre. Il n'est donc plus question seulement de lieu mais de milieu. De plus, si l'esthétique est centrale dans cette approche, c'est en tant qu'expérience sensible du milieu, et non selon la définition plus courante de l'esthétique concernant uniquement la réception de l'œuvre. Il s'agit de considérer la singularité de l'expérience paysagère qui a lieu, en amont du projet, au cours de la création et lors de sa réception. Cette démarche valorisant les relations écologiques et paysagères d'un être vivant et sensible avec son milieu nous fait donc passer d'une esthétique du lieu à une expérience du milieu.

Cette démarche de création inductive, établie dans le cadre d'une approche systémique et expérimentale du projet, implique que le projet commence aussi avec une étude analytique du site. Cette étude vise à repérer les caractéristiques du site, selon différentes approches complémentaires et relationnelles : 
- analyse géographique et naturaliste : géologie, pédologie, hydrologie, climat, faune et flore ;

- analyse géographique anthropique : démographie, aménagement du territoire (habitation, voirie, infrastructures, « espaces verts », exploitation agricole et forestière) ;

- analyse historique : évolution du paysage au cours du temps, patrimoine historique et culturel ;

- analyse perceptive : visuelle, sensorielle, perception et appréciation esthétique ;

- analyse écologique, reliant les différentes relations naturalistes (notamment grâce aux approches synécologique et d'écologie du paysage) et anthropiques ;

Ces différents éléments sont étudiés à différentes échelles, de celle du site à celle de la planète, ce qui permet de les situer dans leurs relations environnementales. Cela se fait par un travail de lecture paysagère (observation sur le terrain), l'étude de documents (cartographiques, historiques...) et les échanges avec les habitants et voisins du site. La prise en compte de l'environnement inclut l'environnement culturel, en occurrence l'histoire et la culture bretonne, et son influence sur les formes du paysage.

L'objectif - artistique et pédagogique - du projet étant de partager une expérience, cette étude de site rationnelle est complétée par une approche plus sensible du terrain. Cette expérience sensible, à la source de la création, se développe d'abord dans un travail de terrain: de découverte, d'observation et d'imprégnation. Concrètement, il s'agit de parcourir le site, marcher et s'arrêter, regarder, écouter, sentir: en bref, d'approfondir une expérience d'immersion d'abord dans ses dimensions corporelles et sensorielles. Il s'agit d'appréhender le paysage comme relation entre un corps et un milieu, la place du corps dans cette démarche artistique consistant à « faire corps avec le paysage » pour «faire le paysage avec son corps ». Les pieds représentent la marche abordée comme démarche de création : il s'agit d'abord de parcourir un lieu pour s'en imprégner, mais aussi de voir comment la marche peut entrainer des modifications du site. Les cinq sens précisent la place du corps dans cette immersion en un lieu : pourquoi et comment voir, toucher, entendre, sentir, goûter un paysage ? On remarque ici l'importance des circonstances particulières de l'immersion (la saison, l'heure du jour ou de la nuit, le temps qu'il fait...). Enfin, l'oeil et la main introduisent une pratique de notation paysagère - par la photographie, le croquis, l'écriture et les relevés (de terre, de végétation...) - pour exposer comment s'effectue, par le corps, le passage de la perception à la présentation du paysage.

Qualifier cette expérience paysagère de «sensible » me permet de relier différentes dimensions de l'expérience : les sensations, la sensibilité (émotionnelle et esthétique) et le sens donné à ce qui est perçu (la signification). Au cours du processus d'immersion, l'expérience corporelle est en fait inséparable d'une expérience émotionnelle et esthétique. Le site perçu est automatiquement mis en relation avec la connaissance intellectuelle que nous en avons, mais aussi avec nos expériences passées. Les dimensions cognitives et émotionnelles influencent directement sur notre perception du paysage. De plus, le site est, plus ou moins consciemment, perçu en relation avec des modèles esthétiques existants. La dimension symbolique du paysage contribue aussi, par la mise en relation des dimensions perceptives, cognitives et émotionnelles, à construire le sens que nous y percevons. Tout cela s'applique au type de paysage concerné (paysage forestier, maritime, marécageux...), aux éléments qui le constituent (arbre, rivière, pierre...) et aux circonstances de l'expérience paysagère (jour ou nuit, saison, climat, luminosité...). L'expérience sensible du paysage se déploie donc à travers divers processus d'évocation, de projection et d'imagination, et c'est ainsi qu'elle « fait sens » pour le sujet de l'expérience.

Dans le cadre d'un projet artistique, cette expérience doit être traduite en signes. Ce qui commence aussi sur le terrain, par un travail de notation, qui prend différentes formes. La notation peut être écrite, par la prise de notes ou l'écriture d'un journal de terrain. Elle peut être visuelle, par la réalisation de croquis cartographiques ou paysagers et la prise de vue photographique. Il peut 
aussi s'agir d'effectuer des relevées, comme des relevés de couleurs, de terre, de végétation, etc. Il s'agit ici d'un travail in situ, réalisé entièrement sur le terrain et dans le moment de l'expérience d'immersion. Ces pratiques de notations sont aussi des formes de médiation, qui entrainent une évolution du statut du paysage. On passe du site au «non-site », expression que Robert Smithson utilisait pour désigner les présentations et représentations d'un paysage dans d'autres lieux et sous d'autres formes: littéraires, graphiques, photographiques et matériologiques. Ces expériences de notations ne se limitent donc pas à des constats sur l'état existant du site, mais impliquent aussi une projection : dans les écrits et croquis apparaissent des idées concernant le projet artistique qui sera mis en place sur le site. On constate que le projet est déjà en germe dans l'observation, ce qui justifie la démarche inductive de création adoptée.

\section{Expériences artistiques et paysagères}

Je vais maintenant vous présenter les réalisations artistiques et les ateliers pédagogiques conçus lors de ce travail de création-recherche, comme des expérimentations visant à partager des expériences écopoétiques.

Je vais commencer par vous présenter mon terrain : le site de Motten Morvan, sur lequel j'ai travaillé de l'été 2016 à l'été 2018. Il se trouve au centre de la Bretagne, sur la commune de SaintAignan, dans une région dont les paysages sont marqués par l'exploitation agricole. Il s'agit d'un terrain de trois hectares, sur les flancs Sud et Ouest d'une colline, qui était en friche depuis quelques années quand je suis arrivée. Ce site était à l'emplacement d'une motte féodale, construite au XVe siècle ; il a ensuite été transformé en une petite propriété agricole. Les paysages sont très diversifiés : il y a une petite ferme traditionnelle (qui tombe en ruine), près de laquelle coule un ruisseau, une petite prairie humide, un ancien verger, un bois, dans lequel se trouvent les vestiges de la motte féodale, et une prairie, dont une partie est régulièrement fauchée, et l'autre, laissée en friche, s'est transformée en saulaie, ainsi que des chemins bordés de haies, le long des champs et habitations voisines. Ce site sur lequel on trouve donc différents milieux écosystémiques constitue en quelque sorte une miniature présentant les divers aspects des paysages de bocage typiques des campagnes bretonnes.

L'objectif de mon projet sur ce terrain était de valoriser la découverte, la connaissance et l'appréciation des différents écosystèmes, en partageant des expériences sensibles de ces paysages. J'ai d'abord privilégié la création d'œuvres et l'animation d'ateliers in situ, afin que que cette expérience soit vécue directement par le public.

\subsection{Réalisations artistiques in situ}

La première création est un sentier paysager, né de la pratique de la marche lors du travail de découverte et d'immersion sur le site. En explorant le site, de manière à découvrir les différents milieux qui le composent, mes pas ont tracé une ligne, que j'ai ensuite élargie à la débroussailleuse. Ce qui a mené à la réalisation d'un sentier paysager, conçu et réalisé de manière à traverser chaque écosystème, ainsi que différents points d'intérêt sur le site. Entretenu par un simple défrichage régulier pendant deux ans, ce parcours permet de visiter tous les milieux caractéristiques de la campagne bretonne. J'ai ensuite conçu trois installations pour ce site (non réalisées à ce jour), dans l'intention de partager avec le public des expériences paysagères inspirées par une approche écopoétique des milieux.

Les «salons sauvages» sont des constructions conçues pour que le marcheur s'y arrête un moment, en s'y asseyant comme dans un salon - mais en extérieur, en pleine nature. Les sièges sont constitués par un ensemble de rondins de chêne disposés en cercle. On s'y assoit en regardant vers l'extérieur du cercle. Il s'agit ici de proposer une approche paysagère singulière, consistant à 
présenter la nature de l'intérieur. Cette installation s'inspire de certaines œuvres d'art environnemental, qui ont pris le contre-pied des approches représentatives du paysage, picturales ou photographiques (qui exposent des images hors de la nature, considérée d'un point de vue extérieur) en proposant une présentation « en direct » des écosystèmes (comme les « sanctuaires » de hermann de vries). Cependant «la nature » y est toujours présentée dans un espace circonscrit sur lequel on pose un regard depuis l'extérieur. L'objectif esthétique des «salons sauvages » est encore plus immersif : la forme circulaire des salons provoque une inversion de la position du spectateur : ce qui est présenté (le paysage) est vu de l'intérieur. Car ce qui apparaît de prime abord comme une installation « au milieu » d'un espace, n'est en fait qu'un prétexte pour présenter le milieu environnant.

L'expérience induite par le fait de s'assoir dans un salon est d'abord une expérience d'immersion, la durée de la pause permettant d'accorder plus d'attention aux sensations et émotions perçues, et donc à la relation établie avec le milieu naturel. Elle est aussi l'occasion de (re)découvrir la vie foisonnante dont sont constitués ces milieux auxquels nous portons rarement attention, et d'observer directement ce que représente la notion de biodiversité (par l'observation des plantes, insectes, oiseaux, etc.). Enfin, il s'agit toujours d'une expérience singulière, qui peut être répétée mais sera toujours différente, selon l'heure, la saison, le temps qu'il fait, etc. Il s'agit donc de partager l'expérience des paysages comme « milieux » de vie dynamiques.

L'intérêt de ces installation, pour instaurer une expérience écopoétique, réside dans leur positionnement dans trois milieux différents : la prairie, la friche et la forêt. Les trois milieux présentent trois niveaux de végétation: les strates herbacée, buissonnière et arborée, qui correspondent aux étapes successives de la végétalisation. Un terrain laissé en friche est d'abord conquis par des herbes, qui poussent au ras du sol et ne montent pas à plus d'un mètre, puis par des ronces, qui protègent les pousses d'arbustes, comme les saules, d'une hauteur entre un et sept mètres, et enfin des arbres, constituant une forêt. La dimension spatiale, c'est-à-dire la hauteur et la forme de la végétation, rend ici visible - et donc immédiatement compréhensible - la dimension temporelle de l'évolution des paysages. L'interêt de ces salons sauvage est aussi de proposer une approche esthétique de la friche, pour en montrer la dimension vivante et biologiquement positive, à l'opposé de la vision traditionnelle de la friche comme ruine agricole, espace abandonné, en cours de dégradation, valorisant ainsi une vision écologique plutôt que productiviste du paysage.

La « cabane-observatoire » est une cabane en bois, conçue pour être construite au point culminant du site, en haut de la prairie de fauche, d'où le regard porte sur les paysages environnant à $360^{\circ}$ (en hiver). Elle est inspirée de la présence sur le site des vestiges de la motte féodale, qui constituait à la fois une habitation et un point d'observation. L'association des modèles de la cabane et de l'observatoire me permet de mettre en forme la corrélation entre observer et habiter le paysage, remettant ainsi en question le présupposé d'un regard extérieur. Il s'agit d'une construction sommaire et de taille réduite, réalisée avec des matériaux simples, extraits du site. En tant qu'observatoire, elle comporte une ouverture dans chaque mur présentant un point de vue privilégié sur le paysage environnant. Ces fenêtres jouent le rôle de «cadre » définissant une portion de paysage, qui est représentée graphiquement à l'intérieur de la cabane - à la manière des tables d'orientation que l'on peut voir sur des chemins de randonnée en montagne. Seulement, ces représentations paysagères montrent les caractéristiques écologiques des paysages observés. La cabane-observatoire propose ainsi une expérience à la fois poétique (liée aux symboliques de la cabane et de l'observatoire), esthétique (vision et représentation paysagère) et pédagogique (découverte de l'écologie). Mais l'essentiel de l'expérience réside dans cette sensation d'habiter le paysage, qui permet d'appréhender autrement notre relation au milieu environnant. Ainsi l'expérience artistique ouvre à une autre manière de voir et d'habiter le monde. 
La troisième installation est plutôt inspirée de ce que l'on appelle le land art, et vise à évoquer des problématiques écologiques globales. Le titre de cette installation : «le foyer », joue sur les deux significations du terme «foyer» : le foyer de cheminée, et par extension le lieu d'habitation, de vie; ou le foyer d'incendie : l'endroit où le feu a démarré, source de la destruction du milieu environnant et donc de mort. L'idée est issue d'une réflexion sur les quatre éléments primordiaux issus de la culture grecque antique : la Terre, l'Air, le Feu et l'Eau. J'ai voulu m'approprier cette approche « élémentaire » pour représenter une vision du monde écologique, en mettant en relation ses dimensions poétiques (la théorie des quatre éléments en tant que système d'analogie unificateur de l'expérience), esthétique (caractéristiques esthétiques, couleurs et matières) et poïétique (matériologie et processus). Les différentes propriétés du feu (matérielles, esthétiques et symboliques) sont mises en œuvre, en relation avec une écopoétique paysagère, pour évoquer de manière sensible la question de la maîtrise de l'énergie et interroger son impact sur nos milieux de vie. La double symbolique du feu, entre création et destruction, associée aux notions d'énergie et de puissance, permettra d'évoquer l'ambivalence de la technologie et les limites de son usage.

Cette œuvre est elle aussi conçue et réalisée dans et pour un milieu singulier, et réalisée à la main à partir de matériaux naturels trouvés sur place. Le milieu choisi pour réaliser cette œuvre est le pied du pylône électrique situé dans la clairière (ancienne basse-cour), le poteau électrique matérialisant la maîtrise de l'énergie et son utilisation abondante dans notre société, et la clairière représentant un exemple concret de modification du milieu par l'action humaine. L'esthétique du feu est abordée comme esthétique de la trace et de la destruction. Le processus poïétique est donc associé à l'utilisation du feu lui-même comme outil de création, par l'action de brûler. Les couleurs du feu seront directement présentes, du rouge-orangé des flammes au noir du charbon, en passant par le gris de la cendre. Le matériau privilégié pour cette œuvre est le bois, forme typique de matériau inflammable. La dimension vitale du feu, comme foyer d'habitation, est représentée par un élément en bois représentant une maison en miniature, placée au pied du pylône électrique. Il s'agit en fait d'une ruche abandonnée, récupérée dans cette clairière même. L'évocation des abeilles, symbolisant l'activité industrieuse et collective, et la fonction d'habitation que remplit une ruche renforcent la signification recherchée. Le fait qu'elle ne soit plus habitée interroge par contre sur la pérennité de ce foyer. L'aspect destructeur du feu, comme foyer d'incendie, est révélé par un cercle de végétation brûlée tout autour de la ruche et du poteau électrique, incluant un saule entièrement noirci par le feu et un tas de cendres.

\subsection{Ateliers d'écoformation par les arts du paysage}

Afin d'approfondir la dimension pédagogique de ce projet et l'implication du public dans un processus non seulement de réception mais aussi de création, j'ai également animé des ateliers pédagogiques, combinant médiation artistique et écoformation.

J'ai imaginé trois ateliers, qui ont ensuite été organisés et animés en partenariat avec le service d'éducation à l'environnement de la Communauté de communes de Pontivy. Les ateliers « Terres de Motten Morvan » et «Les habitants de l'air » faisaient partie d'un programme éducatif annuel, avec un réseau d'écoles privées ayant choisi cette année de développer des activités sur le thème des quatre éléments. L'atelier « Chasseurs de paysage » était intégré au programme des animations d'été, pour la commune de Saint-Aignan, destiné à un public plus large.

Le premier atelier, intitulé «Terres de Motten Morvan », s'est déroulé sur une journée, avec des élèves de maternelle, qui travaille sur le thème de la terre. Le parcours paysager a servi de trame à l'organisation de la journée, autour des différents milieux traversés : la forêt, la friche, la prairie, le ruisseau et la haie. Dans un premier temps, des animations de découverte de la faune et de la flore ont eu lieu dans chaque milieu : observation des terriers de blaireaux et des «petites bêtes » dans l'humus dans la forêt, observation des insectes volants dans la prairie, fabrication de jouets buissonniers avec les branches de saule dans la friche, pêche d'animaux aquatiques dans le ruisseau. 
La deuxième partie de cet atelier est issue de mon propre travail de découverte du site et d'expérimentation artistique, par la pratique du relevé. J'avais ainsi réalisé des relevés de terre et de végétation dans l'idée de présenter les différents milieux par des échantillons, associés à des photos. Les relevés de terre ont été réalisés en frottant une poignée de terre sur une feuille de papier (révélant des différences de couleur et de texture) et ceux de végétation, en disposant des herbes, feuilles ou fleurs sur une autre feuille. L'association de ces deux procédés m'a permis d'imaginer la réalisation d'un carnet d'artiste par les enfants eux-même. La matérialité et le contact direct avec la terre et les plantes en font un procédé adapté aux jeunes enfants, plus sensibles à l'expérience sensible et directe qu'à l'abstraction ou la représentation. Il en résulte un carnet présentant des caractéristiques de différents éco-systèmes tout en témoignant de l'expérience sensible que chaque enfant a vécu dans ces milieux.

Le deuxième atelier, intitulé «Les habitants de l'air », a été réalisé à deux reprises, avec des élèves de CM1 et CM2. La journée a commencée par un «jeu du miroir », activité destinée à enclencher un processus d'immersion : un enfant, guidé par un autre, marche en regardant un miroir placé sous son nez, de manière à voir le ciel (ou les branches des arbres) à la place du sol. Un tour du site en suivant le «parcours paysager » à ensuite été effectué avec l'ensemble des enfants, avec une animation dans chaque milieu, centrée sur la différenciation des écosystèmes et l'observation des «habitants de l'air », c'est-à-dire les animaux volants : écoute des oiseaux dans la forêt, observation des insectes dans la friche, et des oiseaux à la jumelle dans la prairie. Les enfants ont ensuite été répartis en quatre groupes, chacun dans un milieu (ruisseau, friche, forêt, prairie). Les élèves de chaque groupe ont choisi un animal vivant dans ce milieu, puis lequel ils ont construit une « cabane », en utilisant les matériaux trouvés sur place (avec couteau et ficelle comme seuls outils). Puis nous avons refait le tour du site tous ensemble, chaque groupe présentant sa création aux autres. Une vidéo a ensuite été réalisée, présentant ces journées d'atelier.

Le dernier était un atelier photo, intitulé « Chasseurs de paysages », qui a eu lieu en août, sur une durée de trois heures. Quinze personnes y ont participé, la plupart en famille. L'objectif de cet atelier était d'utiliser la pratique de la photographie pour amener le public à observer le paysage et explorer le site avec la plus grande attention, tout en révélant les transformations des milieux naturels dans le temps. J'avais au préalable constitué une série d'une trentaine de photos, prises lors de mes résidences de création sur le site au cours des deux années précédentes. Le choix de ces images a été effectué pour présenter chaque milieu, pour inciter à les observer sous différents angles, pour mettre en valeur des éléments paysagers ayant changé (coupe ou pousse de la végétation, variation de niveau d'eau du ruisseau, clôture ou ouverture de chemin...), et pour valoriser les changements esthétiques des paysages au fil du temps (selon la saison, le temps qu'il fait, l'heure du jour ou de la nuit...). Après un tour du site effectué avec l'ensemble des familles pour leur présenter le terrain et ses limites, un exemplaire de cette série a été confiée à chaque participant, avec la mission de retrouver le lieu exact de la prise de vue, afin d'en réaliser une similaire. Après une heure de « chasse », tout le monde s'est réuni afin de visionner et comparer les photographies. Un dossier photo a été réalisé, réunissant les photographies originales et les recréation de chacun des participants.

\section{Conclusion}

La problématique à l'origine de la préparation de ma thèse en arts plastiques résidait dans la mise en relation entre une pensée de l'écologie et une pratique artistique, afin de voir comment elles pouvaient se développer et s'enrichir l'une et l'autre. La méthode de création-recherche, appliquée à travers le développement d'un projet artistique et de recherche, se montre particulièrement adaptée pour y répondre. Mes réflexions sur l'écologie m'ont amenée a travailler sur le terrain, pour appréhender la relation à un milieu de vie à travers l'expérience sensible vécue. Les expérimentations artistiques m'ont permis d'aller au-delà de l'idée d'une pensée de l'écologie, en 
déployant le concept d'écopoétique dans une approche transdisciplinaire, puis appliquée au paysage. Il en résulte également des résultats de recherches inattendus concernant le rôle de l'art pour l'éducation à l'écologie. En effet, ce n'est pas tant le résultat de la création, c'est-à-dire l'œuvre achevée, que la démarche même de création qui s'est avérée pertinente pour partager une vision du monde écopoétique. Ainsi la recherche par la création artistique sur l'environnement m'a amené à développer des propositions de formation par la création artistique et dans l'environnement.

Pour en savoir plus : www.anaisbelchun.com 